\title{
The Design of System about Cow Activity Based on SVM
}

\author{
Weizheng Shen, Congcong Chen, Shuang Zheng, Shanjun He and Mingda Li \\ School of Electrical and Information, Northeast Agricultural University \\ Harbin, 150030, China \\ wzshen@neau.edu.cn
}

\begin{abstract}
In view of the cow extensive farming, which lacks modern management tools, the cow estrus determine are often laid off. In this paper, a cow behavioral characteristic is designed. The system collects $X, Y, Z$ axis acceleration data through triaxial accelerometer ADXL345, by ZigBee wireless network and RS485 sent to the information center, it's based on the support vector of binary tree to classify their behavior. The result shows, the algorithm for cow's stationary and moving classification accuracy was 93.26\%, a slight and sharp motion accuracy was $84.81 \%$, It provides an effective basis in judging cows in heat or in physical abnormalities. That system contributes to the refinement of cows feeding and health culture, and has great significance for the stable and healthy development of the dairy industry.
\end{abstract}

Keywords: Cows, Activity, ADXL345, SVM

\section{Introduction}

Dairy is an important part of modern agriculture. Stable and healthy development of the dairy industry are significant in aspects of optimizing agricultural structure, increasing farmers' income, improving people's diet structure, enhancing the national constitution, and promoting the development of related industries like national economy. Since the reform and opening up, the dairy industry in China has been rapidly developed. However, for the extensive farming methods and lack of modern management tools, the dairy production keeps rising, the raw milk quality is not high, and the milk yield and conversion is relatively low [1-2]. Using microcontroller and sensor technology to collect key physiological parameters like cow activity rate, it becomes easy to prevent cow disease, accurately determine the cow estrus achieve fine breeding for improving milk yield, and to promote the quality of raw milk.

According to domestic and international studies, it is clear that the estrus activity and health analysis of cows are extremely important physiological indicators. When heat excited, activity levels of cows increase significantly; when cows are sick, their activity will clearly reduce [3]. In China, traditional monitoring methods are mainly used in determining cows' estrus and pregnancy. This method is only suitable for small-scale dairy farms, not for the detection of estrus behavior at night, and could easily lead to chance missing, also cannot meet the growing demand of large-scale farming [4]. Recently, scholars have made many studies on the collection of cows' activity, such as Brehme [5] using ALT pedometer developed computer-aided measurement system to detect cows in estrus; Paula [6] used a three-dimensional accelerometer and support vector machine (SVM) for cow behavior pattern recognition and classification; Nadimi [7] used ZigBee-based mobile wireless sensor networks and artificial neural network to monitor animal behavior and classify their behavior patterns; Yin Order [8] application triaxial accelerometer feature cows classify and predict the 
behaviors of cows in estrus [9]. While domestic studies of such kind started late, which leads to backward technology, so that there is no large-scale application of the product. It is urgent to develop a cow's activity system that fits in China.

\section{System Design}

Considering the application of the system environment for dairy farms, we need to make actual investigation and study about the scale of cow farms, construction, layout and cows living habits before system design. Then we can fully integrate all aspects of a comprehensive design, including to select the wireless network, the node layout, the collision between cows, and the low power consumption [10]. After Comprehensive among comparison Bluetooth, different strengths Wi-Fi, and ZigBee technologies such as infrared, we use ZigBee wireless networks. The major advantage is a short-range, reliable, low complexity, low data rate two-way wireless communication technology, low power, low cost and MANET [11-12]. Because cows body block and other factors, according to the actual test shows that effective communication distance is 60 meters ZigBee barn.

Cows behavioral characteristics classification system structure shown in Figure 1, is composed of activity acquisition nodes, routing nodes, central node, RS485 / RS232 converter and a PC terminal node (information center). Activity collection node wear in cows neck, is responsible for detecting cows three-axis acceleration data; ZigBee wireless network is composed of routing nodes and the central node, the routing node transmits the data to the central node; central node and the through the RS485 RS232 transmit acceleration data to the information center; Information center processes the received data to classify their behavioral characteristics, and cows in heat and physical abnormalities sent to the breeder's phone or PDA via GPRS.

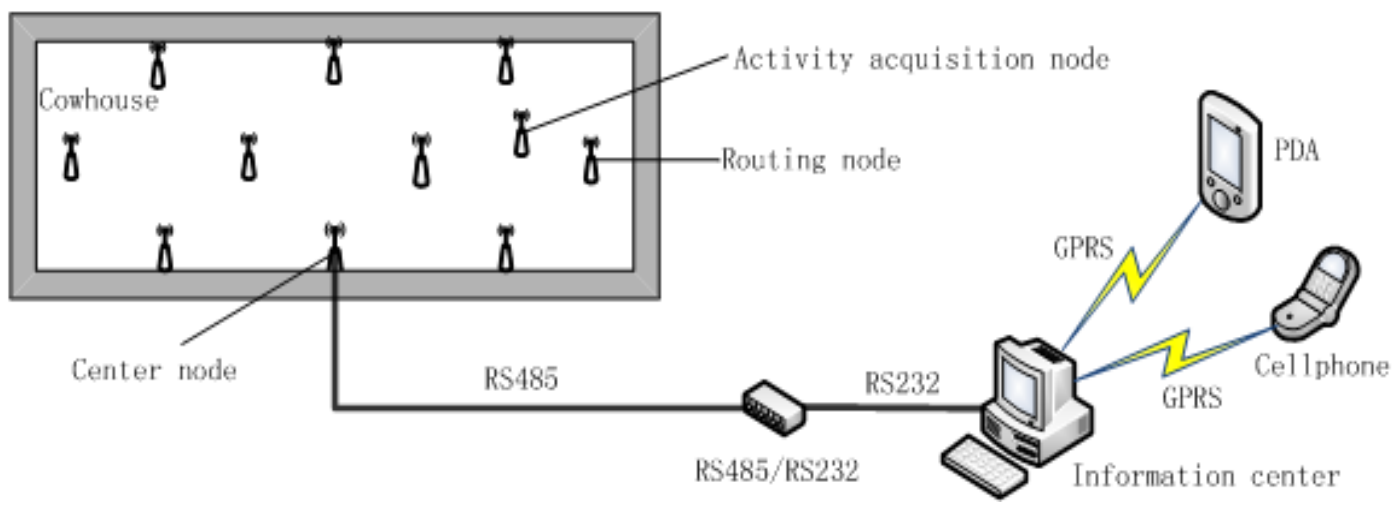

Figure 1. Structure of Cows Behavioral Characteristics Classification System

\section{The Design of Hardware}

\subsection{The Design of Acquisition Activity Node}

Taking into account of the amount of activity nodes need to wear collection in individual cows, in order not to affect the normal physiological activities, the node has to be in a small size, light weight, low power consumption, crash, waterproof and durable. After Comprehensive comparison MSP430, AVR and 8051 microcontrollers, including the different advantages in performance, power consumption and cost and other aspects, the main processor is MSP430F149 microcontroller. It is based on single-chip 16-bit RISC systems, with strong processing capacity and ultra-low power consumption. The operating current of is 
$225 \mu \mathrm{A}$ in active mode, standby only $0.8 \mu \mathrm{A}$ [13]. Triaxial accelerometer uses ADI's ADXL345, and the sensor has low power consumption, small size, high resolution and other characteristics, and the digital output 16 twos complement data format. When the voltage is $2.5 \mathrm{~V}$, the measurement mode as low as $23 \mu \mathrm{A}$, only $0.1 \mu \mathrm{A}$ in standby mode [14]. TI's ZigBee chip selects CC2430 produced in US, combined with a high-performance $2.4 \mathrm{GHz} \mathrm{RF}$ transceiver and a low-power high-performance 8051 microcontroller within the chip; it has a very high receiver sensitivity and robustness; In the receive and transmit mode, current consumption is less than $27 \mathrm{~mA}$, respectively, and $25 \mathrm{~mA}$, only $0.9 \mu \mathrm{A}$ in sleep mode, it very suitable for low power applications. The node structure shown in Figure 2, using two voltage of $3.6 \mathrm{~V}, 1350 \mathrm{mAh}$ lithium battery's capacity. Physical shown in Figure 3, the size of $100 \times 60$ $\times 15 \mathrm{~mm}$.

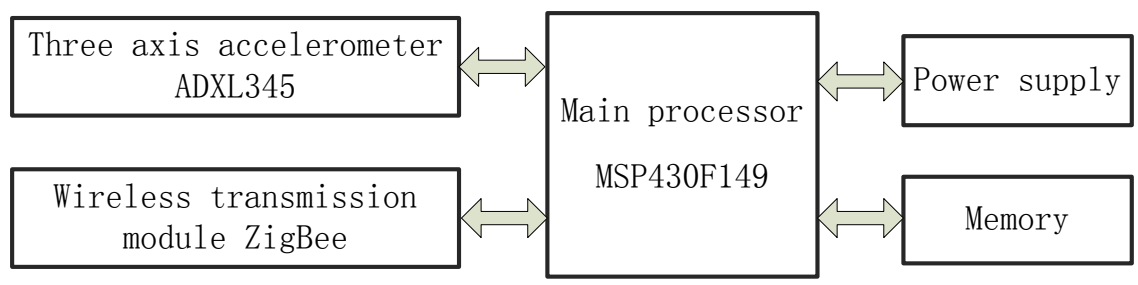

\section{Figure 2. Structure of Cows Activity Data Acquisition Node}

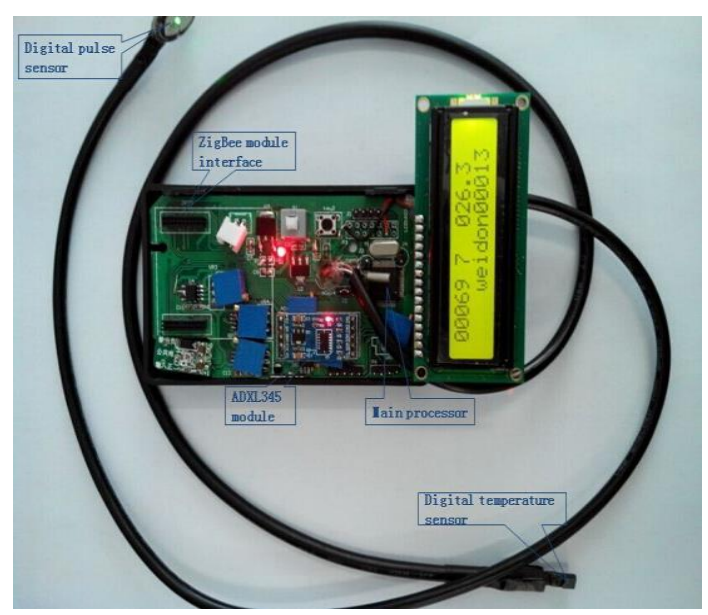

Figure 3. Real Picture of Cows Activity Data Acquisition Node

\subsection{The Design of Central Node and Routing Node}

The central node and the routing node structure are shown in Figure 4 and Figure 5, respectively. Center nodes and routing nodes are used in the production of TI's CC2430 chip. Among them, the central node in the ZigBee network acts as a coordinator, which is responsible for initiating the establishment of the network. The central node and routing nodes need to be fixed, so the main power supply can be $220 \mathrm{~V}$ mains. A $3.6 \mathrm{~V}, 600 \mathrm{mAh}$ lithium battery can be used as standby power supply for maintaining the normal operation of the system when the active power fails. When routing node placement should be considered reasonable in the barn at the bottom of the roof, the effective distance and the height change with cow's activity acquisition node should be considered, according to the actual situation of the barn to minimize routing nodes to reduce costs. 


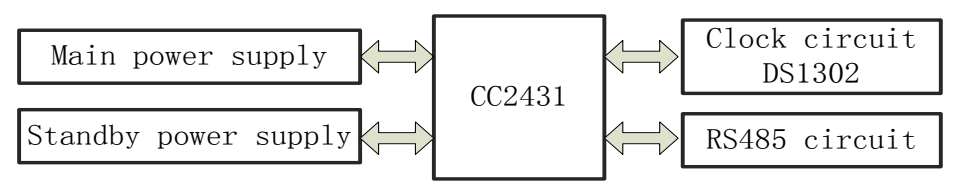

Figure 4. Structure of Center Node

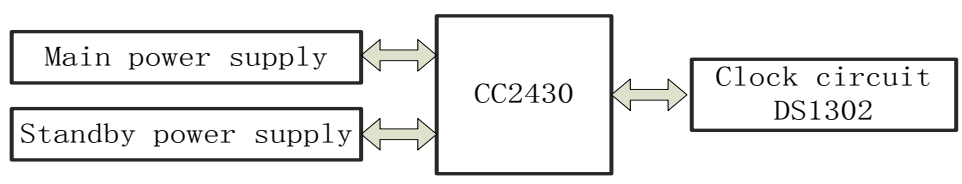

Figure 5. Structure of Routing Node

\subsection{The Design of $\mathrm{RS} 485 / \mathrm{RS} 232$ Note}

Between the central node and Information Center, RS485 is used for data communication. Because RS485 cannot communicate directly with the PC, it needs to be joined with RS485 / RS232 conversion, transferring data to a PC through the serial port. Considering the limited transmission distance of RS485, and different distances between the dairy farm barns and Information Centers, RS485 can be used to extend the transmission distance.

\section{Software Design of System}

\subsection{Program Design of Acquisition Activity}

Acquisition program flow chart of the cow activity as shown in Figure 6. After the device is electrified, it starts to initialize tests and join ZigBee, and then the triaxial accelerometer collects data, by using ZigBee wireless module transmits. After successfully sending the statistics, the machine goes into hibernation, and comes back into normal condition after a certain period of time.

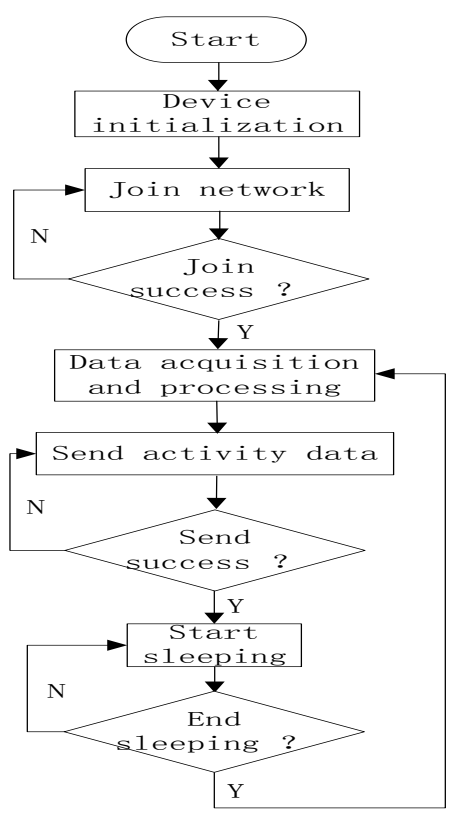

Figure 6. Flow Chart of Activity Data Acquisition Program 


\subsection{Data Collection}

According to different standards and purposes, there are a variety of cows classification methods based on cow behavioral characteristics. In the study of cow habits, Zoologists found that cows will become restless and excited in early estrus, and will be lazy out of estrus. When in heat, the cows usually do creeping behaviors; but when sick, their lying time greatly increase. Therefore, the monitoring is an important indicator to determine the cow behavioral characteristics of estrus and physical abnormality. Cow complicated behavioral cannot be completely distinguish. In order to reduce the difficulty of system modeling, the system will generally be divided into static and movement about cow behavioral characteristics. Among them, static divided into standing and lying, movement subdivided into walking, brisk walking, creeping, jog and run, which is shown in Figure 7.

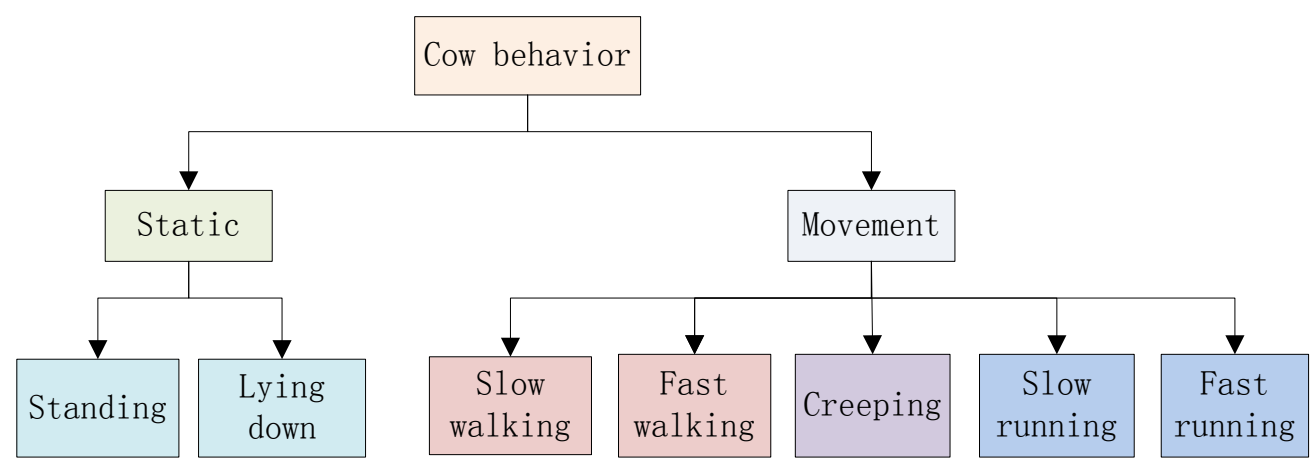

Figure 7. Cow Behavior Classification

The system uses ADXL345 collection through the $\mathrm{X}, \mathrm{Y}, \mathrm{Z}$ axis direction motion to accelerate, with the support vector to classify their behavioral characteristics, in order to characterize their activities. The experiments of data collection happened in the first American Dairy farms in Kedong County by monitoring five bred cattle who wore activity collection nodes for three days. In order to get a three-axis acceleration data under a variety of behaviors, it can provide data support with late cow characteristics classification algorithm. Acquisition node activity should keep pace with the camera time in experiment; you can get behavioral features accurately per second through query time stamp. Acquisition activity node of the design is in collar style, which is wore on the cow's neck. X-axis in ADXL345 three-axis acceleration sensor point cow head, Y-axis and X-axis points to the cow outside on horizontal plane, $\mathrm{Z}$ axis is perpendicular to the point. Acceleration sensor sampling frequency is $10 \mathrm{HZ}$, triaxial data transfer via RS485 and ZigBee wireless network to database and stores in the information center.

\subsection{Design of Model and Algorithm}

As a machine learning method, support vector machine can be trained, which is often used to deal with classification problems. While for multi-class classification problems, support vector machine is also different, in this paper using binary tree structure multi-classes SVM classifier to solve the problem. The idea is divided into two sub-classes of all categories; each class is also divided into two sub-sub-sub-categories, and so on until the final category is divided [15]. The method of constructing multi-class dairy Classifier behavioral characteristics shown in Figure 8. 


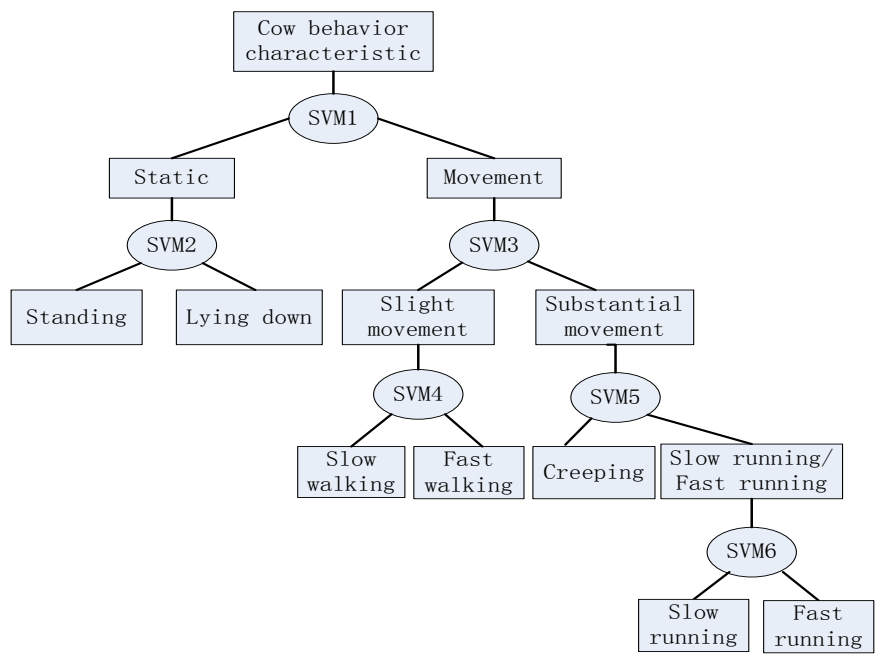

Figure 8. Binary Tree SVM Classification

Firstly, we should construct the SVM mathematical model by using three-axis acceleration data of experiment acquisition. Supposing the sample set like this: Among there,

$x_{i}$ is Input, ${ }^{y_{i}}$ is target output and $n_{\text {is }}$ the number of samples. In this model, the input consists of 19 eigenvectors, such as: the average, maximum, minimum, and average value, standard deviation, correlation coefficients, the absolute value of the maximum. Which is defined as:

$$
x=\left[x_{\text {avg }}, y_{\text {avg }}, z_{\text {avg }}, x_{\max }, y_{\text {max }}, z_{\max }, x_{\min }, y_{\text {min }}, z_{\min }, s m v, x_{s d}, y_{s d}, z_{s d}, c_{x y}, c_{x z}, c_{y z}, x_{a b s}, y_{a b s}, z_{a b s}\right](1) \text {. }
$$

In the formula (1), $\left(x_{\text {avg }}, y_{\text {avg }}, z_{\text {avg }}\right)$ is average for the selected window-axis direction of the acceleration, $\left(x_{\max }, y_{\max }, z_{\max }\right)$ is maximum peak, $\left(x_{\min }, y_{\min }, z_{\min }\right)$ is Minimum peak, $s m v$ is the sum of the average, $\left(x_{s d}, y_{s d}, z_{s d}\right)$ is standard deviation, $\left(c_{x y}, c_{x z}, c_{y z}\right)$ is correlation coefficient, and The maximum of absolute value is $\left(x_{a b s}, y_{a b s}, z_{a b s}\right)$.

Secondly, by selecting the appropriate kernel function to construct optimal hyerplane. Through the above-mentioned SVM model make number of experiments analysis show that select radial basis function as its kernel function, it can achieve better classification results. The core function is defined as:

$$
K\left(x, x_{i}\right)=\exp \left(-\gamma\left\|x-x_{i}\right\|^{2}\right)
$$

where $\gamma$ is nuclear radius parameter. The objective function is

$$
\phi(\omega, \xi)=\min \left\{\frac{1}{2}\|\omega\|^{2}+C \sum_{i=1}^{m} \xi_{i}\right\}
$$

Where is must satisfy the constraints:

$$
\begin{gathered}
y^{(i)}\left(\omega^{T} x^{(i)}+b\right) \geq 1-\xi_{i} \\
\xi_{i} \geq 0, i=1,2, . m .
\end{gathered}
$$

Where, $\omega$ is the weight vector, $\xi_{i}$ is called the slack variable, $C_{\text {is }}$ the parameter control allows error, $b$ is an offset term. Finally, the data processing in MATLAB R2009a. 
Randomly selected $70 \%$ of the data in the database as the training set, the remaining $30 \%$ of data is used as test set. Select the one second time window for processing the data to obtain a set of samples, and match with the corresponding behavior characteristic value to train classifier.

\section{The Results and Analysis of Experiments}

To verify the system performance, a test was taken in the first ranch of Kedong County American Company in July 2014, three cows in heat were chosen as samples. Triaxial accelerometer sampling frequency is $10 \mathrm{HZ}$, loss rate of packet about $5 \%$, the time window is selected for one second, using video surveillance to compare the activity, it should make cows acquisition node time and video surveillance into a consistent time before the test. The number of samples was collected up to10, 248 cow behavioral characteristics classification test results are show in Table 1.

Table 1. Cow Behavior Classification Test Results

\begin{tabular}{|c|c|c|c|c|c|}
\hline \multicolumn{2}{|c|}{ Cow behavior classification } & \multirow{2}{*}{$\begin{array}{c}\begin{array}{c}\text { Number of } \\
\text { samples }\end{array} \\
8132\end{array}$} & \multirow{3}{*}{$\begin{array}{c}\begin{array}{c}\text { Number } \\
\text { of } \\
\text { training }\end{array} \\
7174\end{array}$} & \multirow{3}{*}{$\begin{array}{c}\begin{array}{c}\text { Number of } \\
\text { test }\end{array} \\
3074\end{array}$} & \multirow{3}{*}{$\begin{array}{c}\begin{array}{c}\text { Accuracy } \\
\text { rate }(\%)\end{array} \\
93.26\end{array}$} \\
\hline Static / & $\begin{array}{c}\text { Standing and Lying } \\
\text { down }\end{array}$ & & & & \\
\hline Movement & $\begin{array}{l}\text { Slight movement and } \\
\text { Substantial movement }\end{array}$ & 2116 & & & \\
\hline Static & $\begin{array}{l}\text { Standing } \\
\text { Lying down }\end{array}$ & $\begin{array}{l}5774 \\
2358\end{array}$ & 5692 & 2440 & 72.54 \\
\hline Movement & $\begin{array}{c}\text { Slight movement } \\
\text { Substantial movement }\end{array}$ & $\begin{array}{c}1585 \\
531\end{array}$ & 1481 & 635 & 84.81 \\
\hline $\begin{array}{c}\text { Slight } \\
\text { movement }\end{array}$ & $\begin{array}{l}\text { Slow walking } \\
\text { Fast walking }\end{array}$ & $\begin{array}{c}1043 \\
542\end{array}$ & 1109 & 476 & 69.72 \\
\hline $\begin{array}{l}\text { Substantial } \\
\text { movement }\end{array}$ & $\begin{array}{l}\text { Creeping } \\
\text { Slow running and Fast } \\
\text { running }\end{array}$ & $\begin{array}{l}272 \\
259\end{array}$ & 371 & 160 & 74.86 \\
\hline $\begin{array}{c}\text { Slow } \\
\text { running / } \\
\text { Fast running }\end{array}$ & $\begin{array}{l}\text { Slow running } \\
\text { Fast running }\end{array}$ & $\begin{array}{c}175 \\
84\end{array}$ & 181 & 78 & 70.37 \\
\hline
\end{tabular}

According to the experimental results, the overall performance of the classification model is reasonable. Among them, the classification of still and motion cows is more accurate, reaching 93.26 percent. The slight movement and sharp movement classification accuracy rate is $84.81 \%$. But when cows were subdivided into behavioral characteristics, the classification rate is lower. There are mainly some reasons as the following: Firstly, owing to triaxial accelerometer worn on the neck of the cow, the cow head is more sensitive and moving, leading to the source sample error. Such as cows standing or lying down when at rest, but it will have a slight wobble head occasionally, but we already record as standing or lying down; when cows brisk walking or jogging, the head occasionally sharp swings, also record as brisk walking or jogging. Secondly, due to the behavioral characteristics of dairy cows are artificially observation and judgment, in the case of obvious behavioral characteristics, subdivision two states so that the source sample data more vague. Such as speed between walking and brisk walking or under running jogging and sprints intermediate state. Thirdly, collars on cow neck can slightly move around in a certain arrange, leading to a 
slightly on triaxial accelerometer, affecting cows behavioral characteristics of classification. Fourthly, when the cows in heat creeping, leaping and falling instant acceleration is very large, while little change in residence acceleration and different every creeping duration, can lead to differences in the source of the sample data.

\section{Discussion}

There is still no universal standard for the detection of cow's activity. Their activities are classified mainly by the pedometer through gathering steps and behavioral characteristics. Still, the methods of classifying their behavioral characteristics are different. The system is still not perfect, to be further improved, the next step we will explore the following aspects of the research:

A. Analyzed by gas metabolic analyze cows breathing to calculate it consumes energy, building accelerometer energy equation [16]. With an energy consumption of cows over a period of time to characterize its activity.

B. Activity when collecting node work to be regularly replaced the battery, using a wireless charging method adopted when cow's milk collection node of activity for charging [17], it can avoid frequent replacement battery to extend battery life.

C. Testing on cows of different types and ages, collecting data, optimizing cow behavior features classification algorithm.

D. Increasing cow body temperature and pulse detection module, combined with changes in body temperature and pulse estrus cows, and decided comprehensively cow estrus.

E. Adding cow identification positioning module, reacting fast on physical abnormalities or in estrus, then making an accurate positioning.

\section{Conclusion}

The system completes the acquisition of individual cows' triaxial accelerometer data, transmission, processing, and behavioral characteristics of classification analysis and other functions, and the first ranch in the American Dairy tested. The test results show that the application triaxial accelerometer and support vector function to identify cows various behavioral patterns, but also need to consider how to enhance the quality of the sample data source to improve the classification results. The system uses high-performance, low-power microcontrollers and sensors, stable operation, low development cost, and it has a good application value.

\section{Acknowledgements}

This work was supported in part by the National High-tech R\&D Program of China(863 Program (2012AA101905), Key Technologies R\&D Program of Harbin (2014AB1BN035) and Post-doctoral research starting capital of Heilongjiang province (LBH-Q12146).

\section{References}

[1] L. Shengli, Z. Shengli and L. Jianxin, "2011 dairy industry technology development report", Chinese Journal of Animal Science, vol. 48, no. 6, (2012), pp. 37-44.

[2] F. Fuxiang, X. Jianhua, Z. Yu, etc., "Construction of breeding cows monitoring system", The Journal of Northeast Agricultural University, vol. 43, no. 3, (2012), pp. 32-35.

[3] C. X. Hao, H. S. Qi, M. Gang, etc., "Research activity monitoring technology and its application in dairy cattle breeding management", Chinese cow, no. 8, (2013), pp. 37-40.

[4] L. Yuehua, Y. Yunfeng and F. Jiyun, "Low-power design cow behavior monitor design", The journal of Suzhou University, vol. 30, no. 2, (2010), pp. 27-30. 
[5] U. Brehme, U. Stollberg, R. Holz, et al., "ALT pedometer-New sensor-aided measurement system for improvement in oestrus detection”, Computers and Electronics in Agriculture, vol. 62, no. 1, (2008), pp. 73-80.

[6] P. Martiskainen, M. Jarvinen, J. P. Skon, et al., "Cow behaviour pattern recognition using a three-dimensional accelerometer and support vector machines", Applied Animal Behaviour Science, vol. 119, no. 1, (2009), pp. 32-38.

[7] E. S. Nadimi, R. N. Jorgensen, V. Blanes-Vidal, et al., "Monitoring and classifying animal behavior using ZigBee-based mobile ad hoc wireless sensor networks and artificial neural networks", Computers and Electronics in Agriculture, vol. 82, (2012), pp. 44-54.

[8] Y. Ling, L. Caixing and H. Tiansheng, "Design of cow behavioral characteristics monitoring system based on wireless sensor network", Agricultural Engineering, vol. 26, no. 3, (2010), pp. 203-208.

[9] Z. Dujun, "Research and Design of cow activity monitoring system based on ZigBee technology", Guangzhou: Guangdong University of Technology, (2011), pp. 2-4.

[10] Z. Dujun, B. Hong, L. Zhijian, etc., "Based on ZigBee technology cow activity Acquisition System", Electronic Science and Technology, vol. 24, no. 2, (2011), pp. 44-46.

[11] L. Wenzhong and D. Chaoyu, "ZigBee2006.Wi-Fi and Wireless Location combat", Beijing: Beijing University of Aeronautics and Astronautics Press, (2008).

[12] S. Zhongbin, W. Jin, S. Weizheng, etc., "Cows positioning networking solutions System based on ZigBee technology", The Journal of Northeast Agricultural University, vol. 44, no. 2, (2013), pp. 136-141.

[13] “Texas Instruments Incorporated”, MSP430x1xx Family User's Guide (Rev. F).pdf, (2003).

[14] "Analog Devices",Inc.Three-axis, $\pm 2 / 4 / 8 / 16 g$ digital accelerometer ADXL345 datasheet [EB/OL], (2008), [2013-10-05].http://www.analog.com/static/imported-files/data_sheets/ADXL345.pdf.

[15] Y. Ling, H. Tiansheng, L. Yinghu, etc., "Based on wireless sensor networks support vector machines for cows behavioral characteristics recognition", The journal of Sensing Technology, vol. 24, no. 3, (2011), pp. $458-462$.

[16] X. Jianfeng, "Energy prediction equations motion sensors building and application of students physical activity monitoring", Shanghai: Shanghai Institute of Physical Education, (2011), pp. 2-6.

[17] J. Fachang, "Wireless charging sensor network systems and applications", Hangzhou: Zhejiang University, (2012), pp. 5-10.

\section{Author}

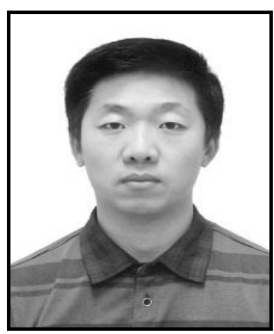

Weizheng Shen, (1977), male, Ph.D., professor, mainly engaged in the research and application of information technology in agriculture 
International Journal of Smart Home

Vol. 9, No. 3 (2015) 